\title{
Endogenous circadian time genes expressions in the liver of mice under constant darkness
}

\author{
Huan Li', Shiyao Zhang', Wenxiang Zhang', Siyu Chen ${ }^{1}$, Anjara Rabearivony', Yujie Shi', Jie Liư ${ }^{2,3}$, \\ Christopher J. Corton ${ }^{3}$ and Chang Liü ${ }^{*}$
}

\begin{abstract}
Background: The circadian rhythms regulate physiological functions and metabolism. Circadian Time (CT) is a unit to quantify the rhythm of endogenous circadian clock, independent of light influence. To understand the gene expression changes throughout CT, C57BL/6 J mice were maintained under constant darkness (DD) for 6 weeks, and the liver samples were collected starting at 9:00 AM (CT1), and every $4 \mathrm{~h}$ in a 24-h cycle (CT5, CT9, CT13, CT17 and CT21). Total RNA was extracted and subjected to RNA-Seq data (deposited as GSE 133342, L-DD). To compare gene oscillation pattern under normal light-dark condition (LD, GSE114400) and short time (2 days) dark-dark condition (S-DD, GSE70497), these data were retried from GEO database, and the trimmed mean of M-values normalization was used to normalize the three RNA-seq data followed by MetaCycle analysis.
\end{abstract}

Results: Approximate $12.1 \%$ of the genes under L-DD exhibited significant rhythmically expression. The top 5 biological processes enriched in L-DD oscillation genes were mRNA processing, aromatic compound catabolic process, mitochondrion organization, heterocycle catabolic process and cellular nitrogen compound mitotic catabolic process. The endogenous circadian rhythms of clock genes, P450 genes and lipid metabolism genes under L-DD were further compared with LD and S-DD. The oscillation patterns were similar but the period and amplitude of those oscillation genes were slightly altered. RT-qPCR confirmed the selected RNA sequence findings.

Conclusions: This is the first study to profile oscillation gene expressions under L-DD. Our data indicate that clock genes, P450 genes and lipid metabolism genes expressed rhythmically under L-DD. Light was not the necessary factor for persisting circadian rhythm but influenced the period and amplitude of oscillation genes.

Keywords: Constant darkness, Circadian time, RNA sequence, MetaCycle, Mouse liver, RT-qPCR

\section{Background}

Organisms adapt and respond optimally to the 24-h lightdark cycles induced by the earth autorotation, and such an adaptation is known as the circadian clock which orchestrates and maintains a 24-h rhythm of mammalian daily activities $[1,2]$. The circadian rhythm is driven by

\footnotetext{
* Correspondence: changliu@cpu.edu.cn

'School of Life Sciences and Technology, China Pharmaceutical University, Nanjing, China

Full list of author information is available at the end of the article
}

circadian clock genes, which are under control of a hierarchical timing system consisting of the master circadian clock located in the hypothalamic suprachiasmatic nucleus (SCN) and a set of peripheral clocks located at the liver and peripheral organs $[3,4]$. The master circadian clock relays temporal information to peripheral clocks through autonomic innervation, glucocorticoids, body temperature and feeding, while peripheral clocks are also influenced by the local metabolic status of the tissues in which they reside $[3,5]$. The core clock is driven by the

(c) The Author(s). 2020 Open Access This article is licensed under a Creative Commons Attribution 4.0 International License, which permits use, sharing, adaptation, distribution and reproduction in any medium or format, as long as you give appropriate credit to the original author(s) and the source, provide a link to the Creative Commons licence, and indicate if changes were made. The images or other third party material in this article are included in the article's Creative Commons licence, unless indicated otherwise in a credit line to the material. If material is not included in the article's Creative Commons licence and your intended use is not permitted by statutory regulation or exceeds the permitted use, you will need to obtain permission directly from the copyright holder. To view a copy of this licence, visit http://creativecommons.org/licenses/by/4.0/ The Creative Commons Public Domain Dedication waiver (http://creativecommons.org/publicdomain/zero/1.0/) applies to the data made available in this article, unless otherwise stated in a credit line to the data. 
transcriptional activators including Clock, Bmal1 (Arnt1) and Npas2, which heterodimerize (CLOCK/BMAL1, NPAS2/BMAL1) and bind to DNA sequences in the promoters of regulated genes called E-box response elements $[5,6]$. BMAL1/CLOCK drives the transcription of several distinct negative feedback genes, including two Cryptochrome (Cry1, Cry2) genes and three Period genes (Per1, Per2, Per3), whose products multimerize and suppress the activation of the CLOCK:BMAL1 complex. The CLOCK/ BMAL1 heterodimer also robustly governs the circadian expression of nuclear orphan receptors Rev-erba (Nr1d1), the PAR-bZip family members $D b p$ and other clock targeted genes [6,7].

The circadian clock, as a rhythmic epigenomic programmer, controls a group of metabolic processes, such as the sleep/wake cycle, the fasting/feeding cycle, glucose homeostasis, as well as lipid and bile acid metabolism $[2,8]$. The crosstalk between circadian clock and metabolism is known and the disorder of circadian clock destroys metabolic homeostasis [8-11]. For example, Clock mutant mice developed hyperglycemia, hypo-insulinemia, and obesity [12]. Specific knockout of clock gene in liver resulted in hyperglycemia [13]. Deletion of the Rev-erbo gene decreased bile acid synthesis and reduced bile acid accumulation in the liver [14]. In addition, the rate-limiting enzymes of some metabolic processes such as Cyp $7 a 1$ were regulated by circadian clock genes [9]. Taken together, the crosstalk between circadian clock and metabolism is vital in maintaining metabolic homeostasis [10].

Circadian rhythms are generated by intrinsic oscillation of their specific central clock [15]. Light is a conspicuous zeitgeber for the circadian system (Zeitgeber is defined as a rhythmically occurring phenomenon acting as a cue in the regulation of body's circadian rhythms). However, under the constant light or dark conditions, a rhythm still persists [16]. Three models are often used to perform circadian researches: light-dark conditions $(12 \mathrm{~h}$ light:12 $\mathrm{h}$ dark, LD), constant darkness (DD), and constant light (LL) [17, 18]. Although most clock researches are carried out using the LD model, several studies described circadian rhythm under the DD condition where the time is referred as CT units, different from Zeitgeber Time (ZT) [17, 19-21].

In the DD model, animals are shielded from light to eliminate entrainment effects on the circadian clock, known as "free running rhythm", which is regarded as mammalian biological signal in metabolism [18, 22]. Under DD condition, wheel-running experiment was performed to monitor the rhythm of locomotor activity [23]. It has been reported light and darkness had acute effects on the activity and temperature rhythms of a subterranean rodent, the Anillaco tuco-tuco [24]. Besides, aberrant emotional behaviors and cognition were related to altered light conditions [25]. The composition of the murine gut microbiome, memory Function, and plasma metabolome was influenced by DD [26]. Under the DD model and fast-refeeding conditions, we have identified the Angptl8 as a hepatokine that mediates food-driven resetting of hepatic clock and diurnal rhythms of metabolic genes in mice [27]. Therefore, DD is an important factor not only for circadian clock but also for metabolism. To examine the circadian rhythm of circadian clock genes, P450 enzyme genes, and lipid metabolism genes under DD condition would benefit and impact the metabolism research.

The present study was conducted in mice acclimated to DD conditions for 6 weeks (called long time darkdark condition, L-DD) to eliminate light influence. The liver samples were collected based on CT time points and subjected to RNA sequencing to profile the changes of gene expression abundance in the liver [27]. MetaCycle was used to screen the oscillation genes and realtime RT-qPCR was used to confirm the gene expression patterns. To further understand the endogenous rhythm of oscillation genes in metabolism, comparisons of P450 enzyme genes, lipid metabolism genes, and clock genes under LD, L-DD and shorter (2 days) constant darkness (S-DD) were made. Our results clearly demonstrate that the endogenous circadian rhythm of clock genes, P450 genes and lipid metabolism genes robustly persists under L-DD. The oscillation patterns were similar but the period and amplitude of those oscillation genes were slightly altered. Light was not the necessary factor for persisting circadian rhythm but influenced the period and amplitude of oscillation genes.

\section{Results}

The RNA-sequencing data of L-DD was first trimmed mean of M-values (TMM) normalized, followed by MetaCycle analysis to identify oscillation genes. The results showed that $12.1 \%$ of the genes under L-DD exhibited a statistically significant $(P<0.05)$ rhythmically expression (Additional file 1). These oscillation genes were visualized in the heatmap (Fig. 1a). To further understand the functional and biological pathways of those oscillation genes, Gene ontology (GO) and Kyoto encyclopedia of genes and Genomes (KEGG) analysis were performed. For the GO (biological processes) analysis, the top 5 enriched numbers of L-DD oscillation genes were mRNA processing, aromatic compound catabolic process, mitochondrion organization, heterocycle catabolic process and cellular nitrogen compound mitotic catabolic process (Fig. 1b). The KEGG analysis showed that the oscillation genes under L-DD were involved in the circadian rhythm (Fig. 1c).

In order to compare the gene oscillation patterns under L-DD in the present study, with those under LD and SDD, the RNA-sequence data of LD (GSE114400) [28] and 
A

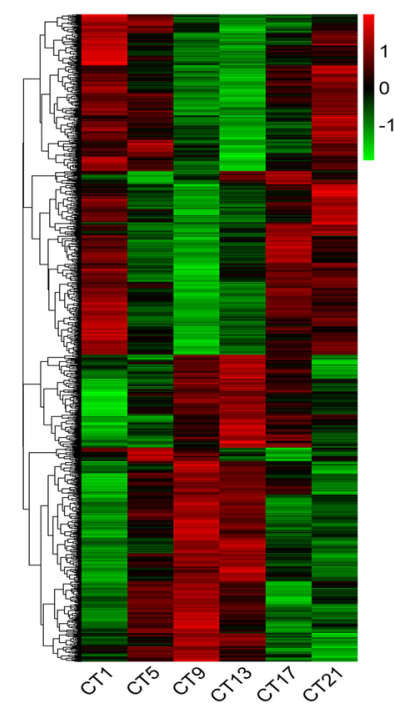

B

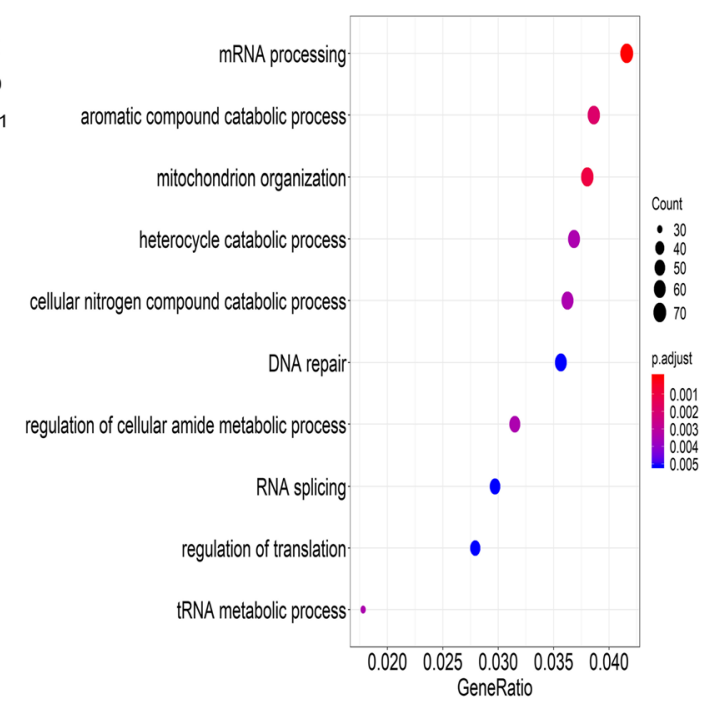

C

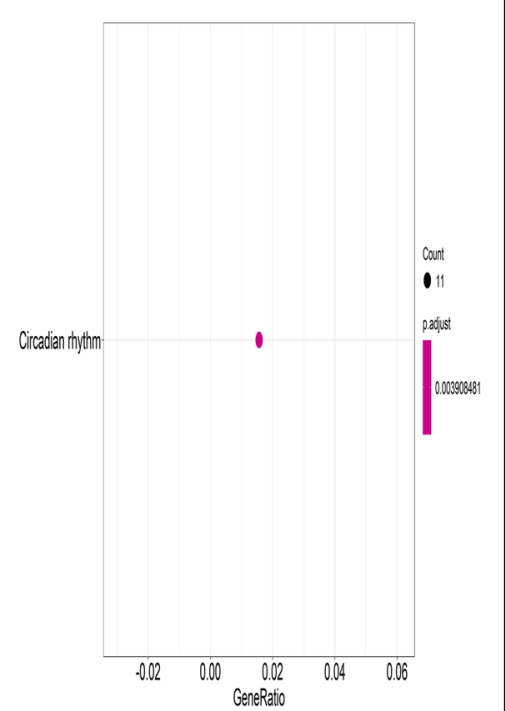

Fig. 1 Oscillation genes profile and biological function analysis under L-DD. RNA-Seq data under L-DD was normalized by TMM and subjected to MetaCycle to screen the oscillation genes. GO (Biological process) and KEGG were performed to analyze the biological function of those oscillation genes. a Heatmap displaying oscillation genes under L-DD. b GO analysis of oscillation genes under L-DD. c KEGG analysis of oscillation genes under L-DD

S-DD (GSE70947) [29] were retrieved from the GEO database and subjected to the MetaCycle analysis after the same TMM normalization. The raw filtered results were listed in additional file 2 and additional file 3, respectively. The oscillation gene patterns of LD were shown in Fig. 2a. GO analysis showed the oscillation genes under LD were enriched in the ribonucleotide metabolic process, mitochondrion organization and fatty acid metabolic process (Fig. 2b). KEGG analysis revealed that those oscillation genes were enriched on retinol metabolism, steroid hormone biosynthesis and drug metabolism-cytochrome P450 pathway (Fig. 2c). The oscillation gene patterns

\section{A}

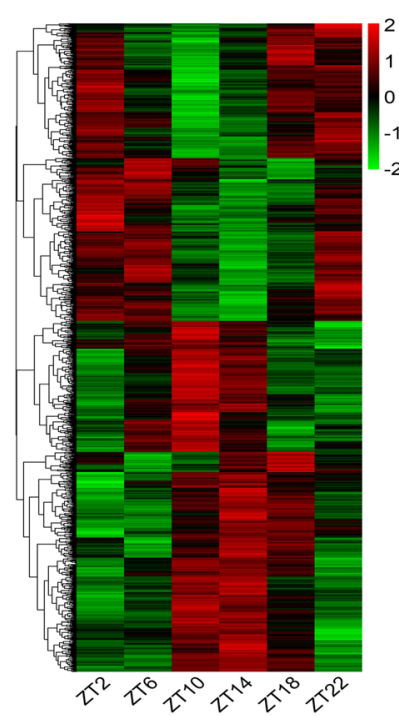

B

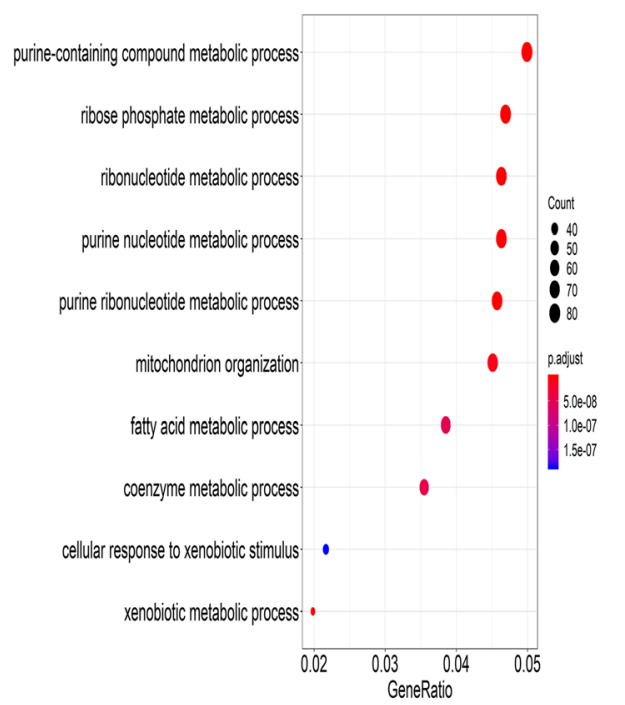

C

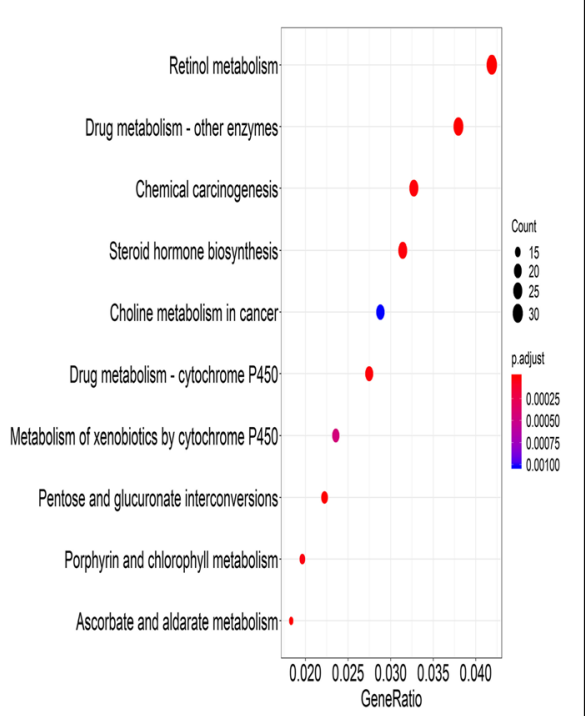

Fig. 2 Oscillation genes profile and biological function analysis under LD. RNA-Seq data under LD was normalized by TMM and subjected to MetaCycle to screen the oscillation genes. GO (Biological process) and KEGG were performed to analyze the biological function of those oscillation genes. a Heatmap displaying oscillation genes under LD. b GO analysis of oscillation genes under LD. c KEGG analysis of oscillation genes under LD 


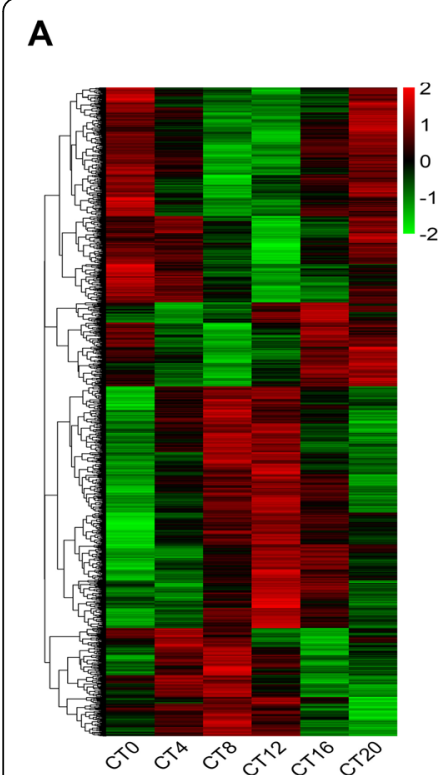

B

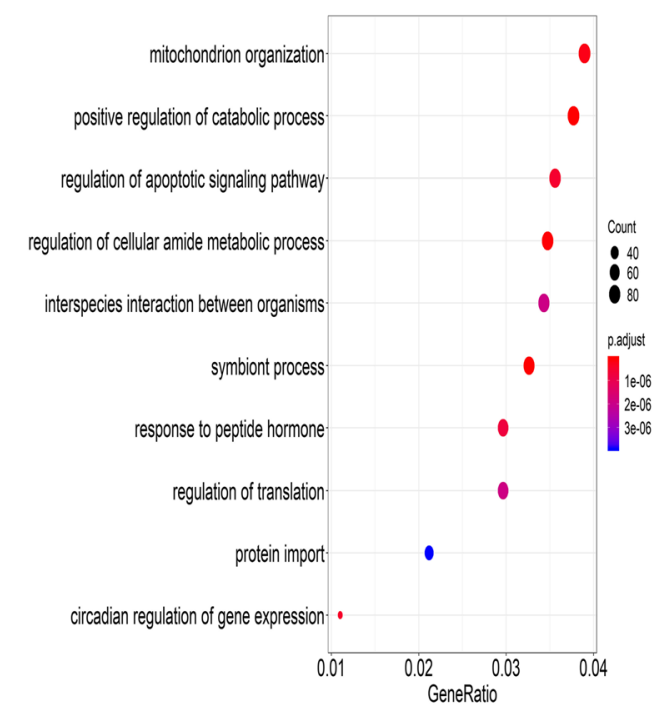

C

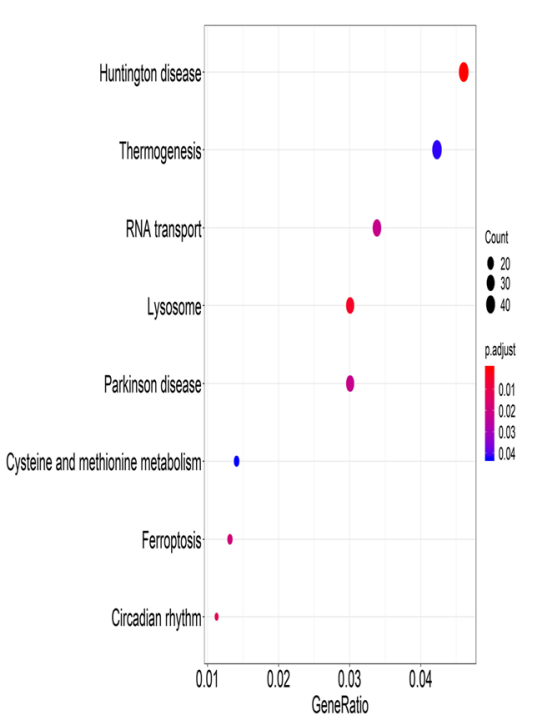

Fig. 3 Oscillation genes profile and biological function analysis under S-DD. RNA-Seq data under S-DD was normalized by TMM and subjected to MetaCycle to screen the oscillation genes. GO (Biological process) and KEGG were performed to analyze the biological function of those oscillation genes. a Heatmap displaying oscillation genes under S-DD. b GO analysis of oscillation genes under S-DD. c KEGG analysis of oscillation genes under S-DD

under S-DD were shown in Fig. 3a, The oscillation genes under S-DD were mainly gathered at mitochondrion organization, catabolic process, apoptotic signaling pathway and cellular amide metabolic process with GO analysis (Fig. 3b). KEGG analysis illustrated those oscillation genes participated in Huntington disease, Thermogenesis, RNA transport and Lysosome pathway (Fig. 3c). It is clear that under L-DD conditions, GO was mainly enriched in catabolic metabolism, and KEGG was enriched in circadian rhythm, which was quite different from LD, and somewhat different from S-DD.

To further study the characteristics of oscillation genes under L-DD, the oscillation genes under three different light conditions of LD, S-DD and L-DD were compared. As shown in Venn diagram (Fig. 4a), there are 1779 hepatic oscillation genes under LD condition (1779/ $\left.14498^{*} 100=12.3 \%\right), 2483$ under S-DD condition (2483/ $13522 * 100=17.9 \%)$ and 1763 under L-DD condition $\left(1763 / 14518^{*} 100=12.1 \%\right)$. Comparison of the oscillation genes between LD and S-DD showed that 458 genes expressed rhythmically both at LD and S-DD. Three hundred eighty-seven genes oscillated at both S-DD and L-DD. Three hundred three genes oscillated at both LDD and LD. Notably, 114 genes persisted oscillating at all the three conditions, suggesting their oscillations were endogenous. GO analysis revealed that the 114 genes were involved in ribose phosphate metabolic process, purine-containing compound metabolic process, rhythmic process, fatty acid metabolic process and purine ribonucleotide metabolic process (Fig. 4b).
To better understand the oscillation pattern under LDD, Fig. 5 showed that the circadian clock genes exhibited typical oscillation patterns. Table 1 listed the period and amplitude of circadian clock genes under LD, S-DD and L-DD. Compared to LD and S-DD conditions, the periods of clock core genes Bmal1, Clock and Npas2 got longer but the amplitudes were slightly decreased. For period genes Per1 and Per2, L-DD significantly increased their period but did not influence the period of Per3. The period of Cryl gene got longer under L-DD but the amplitude was unaltered. For Cry 2 gene, its period became shorter but the amplitude was increased under LDD. L-DD had no influence on period of Rev-erb $\alpha$ but increased its amplitude, on the contrary, increased period of Rev-erb $\beta$ was observed under L-DD. For clocktargeted genes, compared to LD condition, the period of $D b p$ decreased but the period of Tef increased. The amplitudes of $D b p$, Tef and $H l f$ all increased under L-DD.

Cytochrome P450 (CYP450) enzymes are the key enzymes in the liver involved in the metabolism of drugs, steroids, vitamins, and other chemicals [30]. CYP450 superfamily is typical to represent most common phase I drug-metabolizing enzymes [31]. For example, CYP1CYP3 members in the CYP450 families are responsible for the phase I-dependent metabolism of $70-80 \%$ clinically used drugs [32]. In addition, our previous study has reported that some of the CYP450 genes were rhythmically expressed in the mouse liver under LD condition [33]. The presented study illustrated that CYPs exhibited robust circadian rhythm in mouse liver under L-DD. 


\section{A}

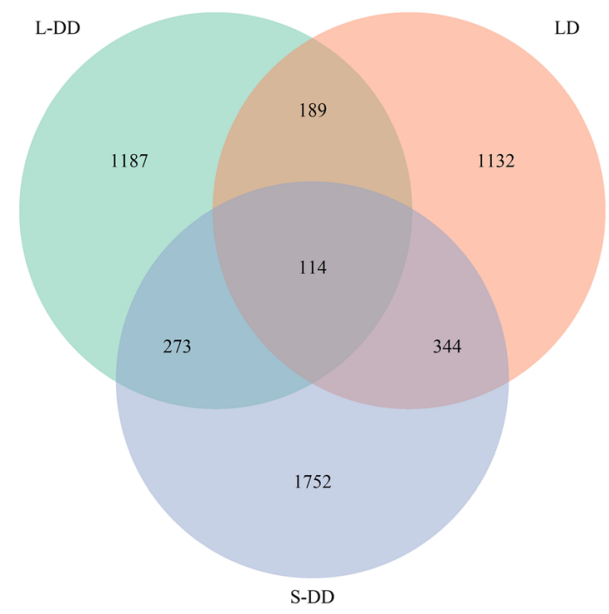

B

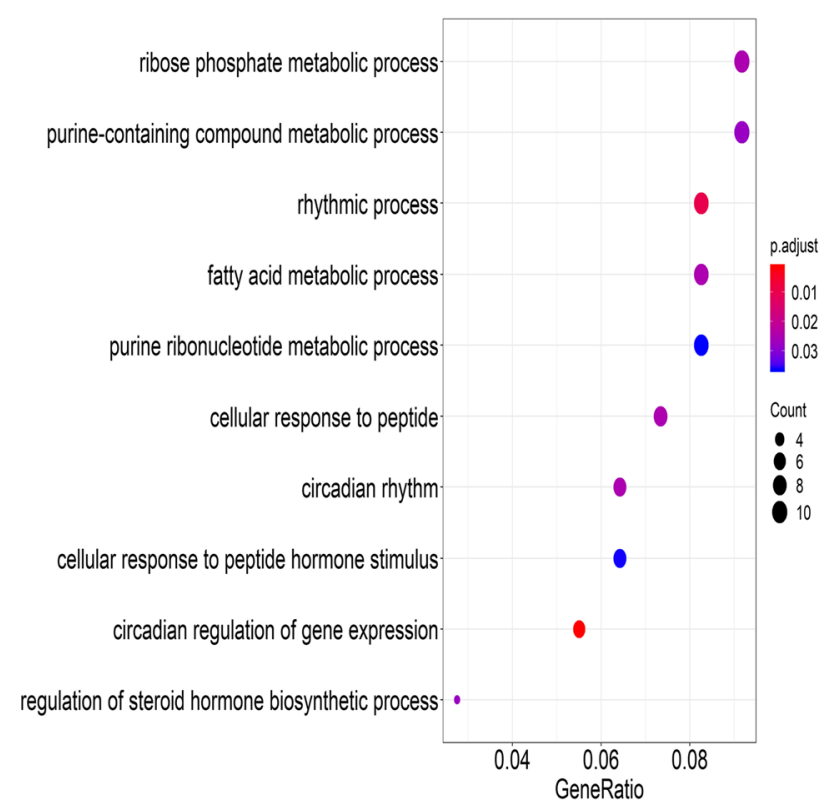

Fig. 4 Comparisons of hepatic oscillation genes under LD, S-DD and L-DD. a Venn diagram displaying the overlapping number of oscillation genes under LD (red), S-DD (green) and L-DD (blue). b KEGG analysis of overlapping genes under three conditions

Figure 6 showed the oscillation pattern of some P450 genes with typical circadian rhythm. Table 2 presented the periods and amplitudes of those oscillation CYPs genes under LD, S-DD and L-DD. Cytochrome P450 families 1-3 (CYP1-CYP3) are involved in drug and steroid metabolism [30]. Compared to the LD and S-DD, the periods of Cyp1a1, Cyp2a4 and Cyp2d40 decreased but for Cyp2a5, Cyp2b10 and Cyp2c29, their periods were increased under L-DD. L-DD extended the period of Cyp2e1 but had no influence on the period of Cyp2g1. Cyp $4 a 14$ is responsible for fatty acid metabolism, and its period was downregulated under L-DD. Cyp7a1 is involved in bile acid biosynthesis [34], and Cyp51 is an essential enzyme in sterol biosynthesis [35]. Periods of the two genes significantly increased under L-DD. Compared to LD and S-DD, L-DD failed to alter amplitudes of most gens including Cyp2a5, Cyp2c29, Cyp2d40, Cyp2e1, Cyp51 and Cyp7a1. However, for Cyp1a1, Cyp2g1 and Cyp4a14, their amplitudes were increased. The amplitudes of Cyp $2 a 4$ and Cyp $2 b 10$ were decreased under L-DD.

The oscillation genes that regulate cholesterol, lipid and fatty acid metabolism are shown in Fig. 7 and Table 3. Hmgcs 1 encode the enzyme HMG-CoA (the substrate of $\mathrm{Hmgcr}$ ) and play an important role in cholesterol metabolism [36]. Compared to LD, the period of Hmgcs 1 increased $5 \mathrm{~h}$ but its amplitude had no change under LDD. Acyl-CoA thioesterase (Acot) genes act as auxiliary enzymes in the $\alpha$ - and $\beta$-oxidation of various lipids in peroxisomes [37]. Compared to LD, the period of Acot3, Acot4, Acot8 slightly decreased under L-DD. For the amplitude, L-DD increased the amplitude of $A \cot 3$, and Acot4 but had no influence on Acot13 and Acot8. Lipin1, Lipin2 and Thrsp are involved in lipogenesis [38]. Compared to LD, the period of Thrsp gradually decreased but amplitude increased under S-DD and L-DD. On the contrary, the periods of Lipin1 and Lipin2 were increased under L-DD. Stearoyl-Coenzyme A desaturase (Scd) genes encode the key enzymes involved in the conversion of saturated fatty acids into monounsaturated fatty acids [39]. L-DD decreased the period of $S c d 1$ and Scd2. The recent research illustrated Angptl3 and Angptl4 played important roles in lipid metabolism [40]. The presented study found that the expression of Angptl3 and Angptl4 showed circadian rhythm under LD and L-DD. Compared to LD, the period of Angptl3 became long under L-DD.

It has been reported that several factors influenced the identification of RNA-seq oscillation genes, including: (1) number of time points and replicates, (2) choice of analysis algorithm, (3) method of read-depth normalization, (4) number of reads per sample, and (5) choice of statistical analyses. It should be noted that the computational approach is an expedient to generate synthetic test data, rather than an approach to identify bona fide cycling transcripts [41]. In order to verify the results of MetaCycle, RT-qPCR was performed on selected genes of our interest (Fig. 8). The expression of Acot3 and Acot4 oscillated 


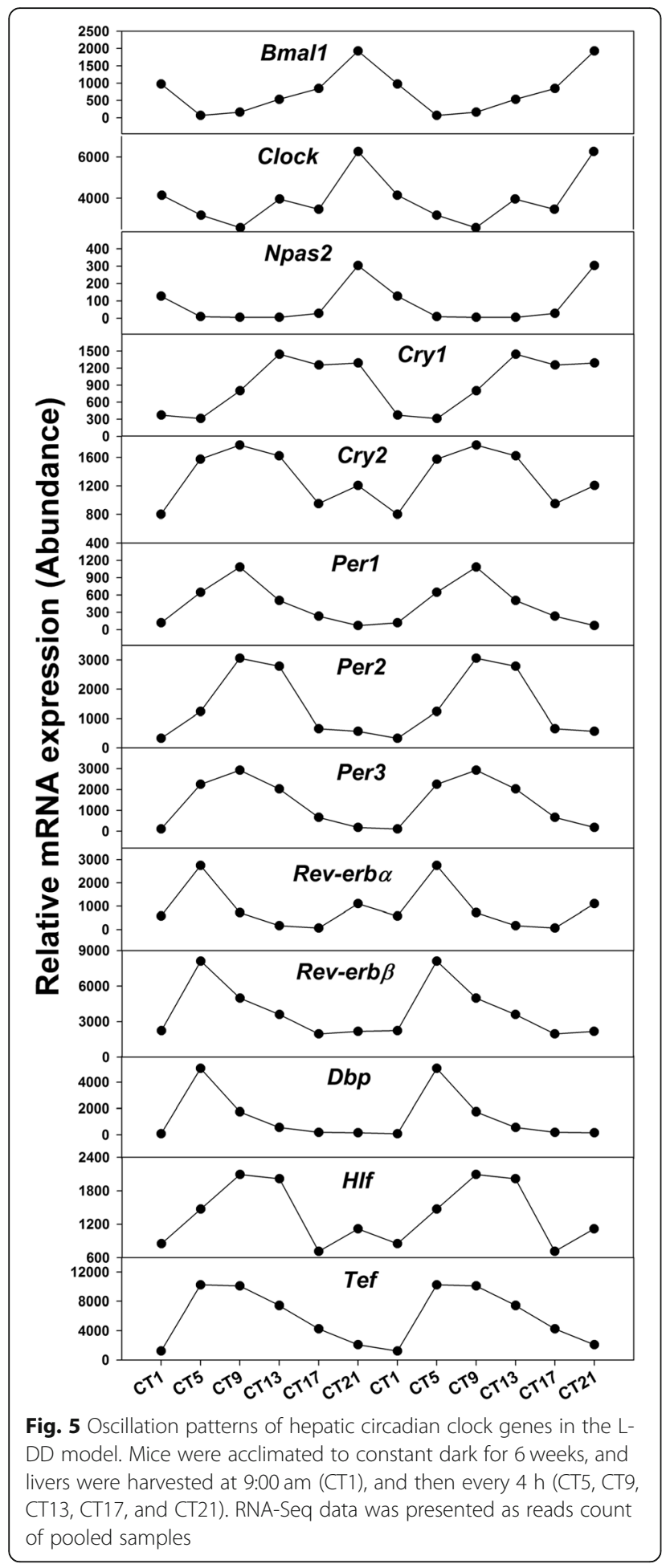

rhythmically at $24 \mathrm{~h}$ cycle of mouse liver. Both highest expressions occurred at CT13, the amplitudes were 2.5 and 5 for Acot3 and Acot4. Scd1 and Scd2 also exhibited robust rhythm. The expression of $S c d 1$ peaked at CT21 and nadir at CT2. For $S c d 2$, the highest expression occurred from CT13 to CT21 and the lowest expression at CT2.
Besides, Hmgcr and Usp 2 also displayed robustly oscillation and their expression peaked at CT13 and CT9 respectively.

\section{Discussion}

The present study extended our recent publication [27], and screened the oscillation gene in mouse liver under L-DD through MetaCycle. Approximatively $12.1 \%$ of the genes exhibited $24 \mathrm{~h}$ oscillation under L-DD. The KEGG and GO analysis further revealed circadian significance of oscillated genes. The oscillation genes under L-DD were enriched in the circadian rhythm pathway. The endogenous circadian rhythm of clock genes, P450 genes and lipid metabolism genes under L-DD were further compared with LD and S-DD. The oscillation patterns were similar but the period and amplitude of those oscillation genes were slightly altered. This is the first study to profile CT gene expressions under L-DD, indicating that the circadian rhythm of clock genes, P450 genes and lipid metabolism genes still robustly persists under L- DD. Light was not the necessary factor for persisting circadian rhythm but influenced the period and amplitude of oscillation of metabolism genes.

Consistent with the literature where animal circadian rhythms persist under constant darkness [42], the present study revealed that when mice are housed in constant darkness for up to 6 weeks, hepatic circadian rhythm still persists. Oscillation genes under LD, S-DD and L-DD were enriched in different biological process respectively, which was caused by an altered light cycle. As shown in Venn Diagram, more than $60 \%$ of oscillation genes kept rhythmicity only in their unique light cycle, once the light cycle changed, their oscillation

Table 1 Comparisons of hepatic circadian clock genes under LD, S-DD and L-DD conditions

\begin{tabular}{|c|c|c|c|c|c|c|c|c|c|}
\hline \multirow[t]{2}{*}{ Name } & \multicolumn{3}{|c|}{$\boldsymbol{P}$-value } & \multicolumn{3}{|c|}{ Period (hour) } & \multicolumn{3}{|c|}{ _rAmplitude } \\
\hline & LD & S-DD & L-DD & LD & S-DD & L-DD & LD & S-DD & $L-C$ \\
\hline 1 & 0.07 & 0.00 & 0.28 & 21.56 & 22.06 & 22.47 & 0.51 & 0.68 & 0.50 \\
\hline Un & 0.01 & 0 & 0 & 3 & 3 & 15 & 0.08 & 3 & 0.06 \\
\hline as & 2 & 0.00 & 0.0 & 22.06 & 21.14 & 24.45 & 2.25 & 3.86 & 2.50 \\
\hline & 0.05 & 0 & 0 & 0 & 2 & 6 & 0.62 & 0. & 0.95 \\
\hline 2 & 0.08 & 0.01 & 0.00 & 20.16 & 22.95 & 21.03 & 0.39 & 0.42 & 0.40 \\
\hline 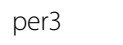 & 1 & 0.00 & $0 . c$ & 3 & 23 & 22.86 & 0.29 & 0.4 & 0 \\
\hline ry1 & 0.17 & 0.02 & 0.04 & 21.59 & 23.05 & 23.31 & 0.28 & 0.30 & 0.26 \\
\hline $\begin{array}{l}y< \\
\text {. }\end{array}$ & 0. & 0.14 & 0.04 & 4 & 8 & 5 & 8 & 0.06 & 0.15 \\
\hline v-erba & 0.17 & 0.04 & 0.15 & 21.97 & 22.55 & 21.33 & 0.64 & 0.65 & 1.07 \\
\hline Rev-erb $\beta$ & 0.0 & 0 & 0. & 9 & 2 & 22 & 0.16 & 0. & 0 \\
\hline bp & 0.02 & 0.00 & 0.24 & 22.11 & 20.90 & 20.61 & 0.46 & 0.95 & 0.85 \\
\hline Tाटा & 0.0 & 0.00 & 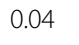 & 22.73 & 21.70 & 0 & 0.13 & 0.19 & 0 \\
\hline If & 0.04 & 0.02 & 0.02 & 20.12 & 22.34 & 20.02 & 0.11 & 0.06 & 0.19 \\
\hline
\end{tabular}

Note: 0.00 indicates that the values are less than 0.004 


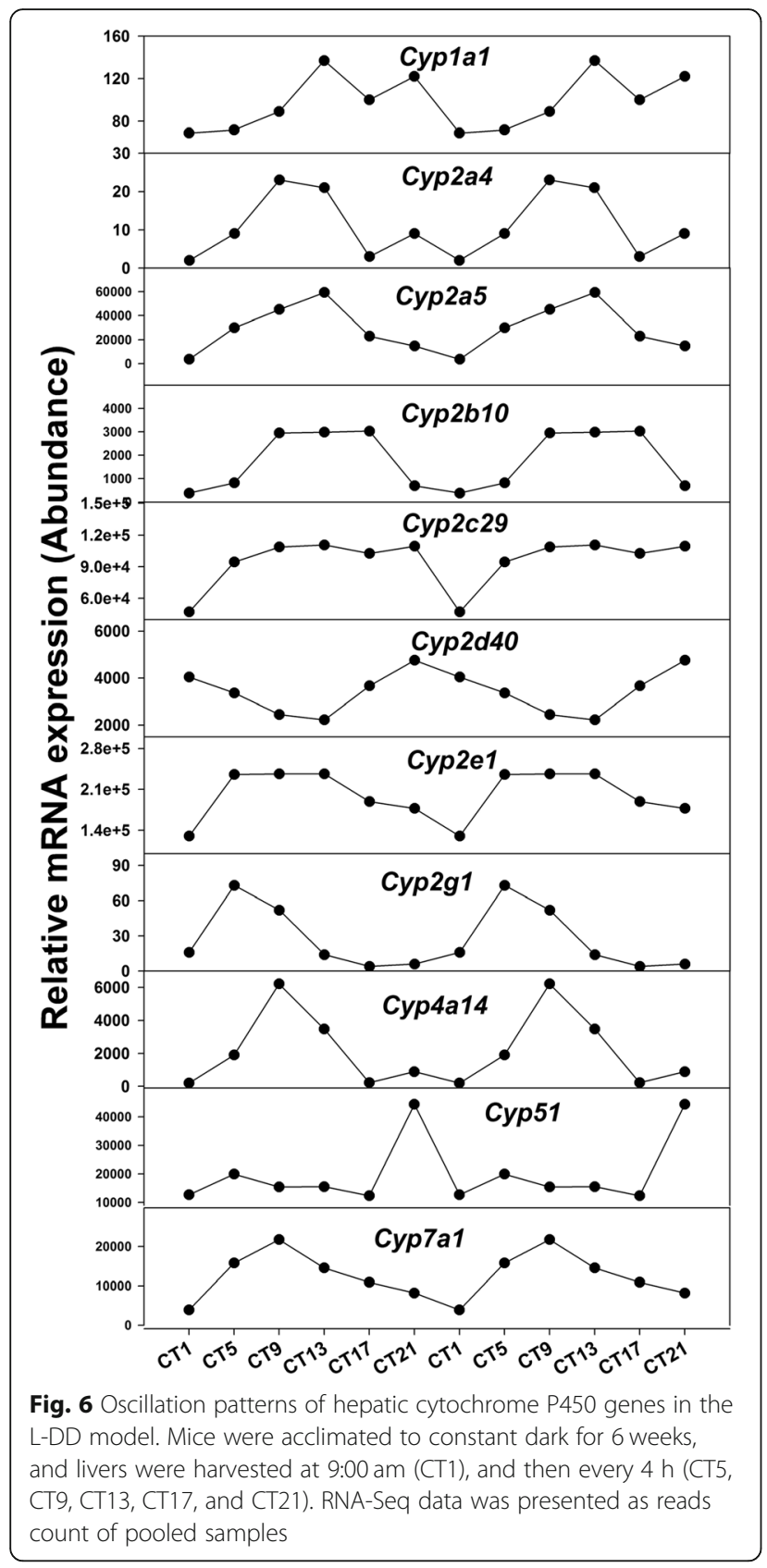

disappeared. For example, circadian rhythm of reactive oxygen species (ROS) levels in Daphnia pulex are changed in different light condition [43]. The mRNA expression of Connexin30 and Connexin43 in mouse SCN exhibit oscillation under LD condition but disappeared in DD and LL [44]. Therefore, the oscillation of some genes depends on the entrainment of light while the others are endogenous.

For circadian clock genes, they displayed robust rhythm under L-DD conditions. Their oscillations were endogenous and knock out of these genes resulted in the loss of rhythms. For example, under DD conditions for 8 days, all wild-type mice remain rhythmic, but $2 / 3$ of Clock mutant mice are arrhythmic [45]. Mice deficient in both mPer1 and mPer2 do not express circadian rhythms [46]. In 2weeks DD conditions, Rev-erb $\alpha$ and Rev-erb $\beta$ retain dynamic oscillation throughout the 24-h cycle both in the SCN and liver [19], and when liver-specific knocks out Rev-erb $\alpha$ and Rev-erb $\beta$ genes, the circadian rhythm of bmal1, clock and output genes are all lost [47]. Therefore, light cycle is not necessary for endogenous circadian rhythm but influences the period and amplitude of oscillation genes. As shown in Table 1, L-DD lengthened the cycle of most circadian clock genes. Lack of light entrainment could explain why the period of most circadian clock genes became longer under L-DD.

In the present study, the P450 superfamily genes also exhibited robust circadian rhythm under DD conditions, and the peak of those oscillation genes occurred at the time of transition from light to dark. P450 genes are involved in metabolism of drug, xenobiotics, steroids and fatty acids [30]. It has been reported that circadian oscillators orchestrate the circadian rhythm of CYPs, for example, $D B P$ regulates the circadian expression of CYP2A, 7A [48]. ROR $\alpha / \gamma$ regulates the rhythm of CYP2B, 4A $[49,50]$. CREM modulates the circadian expression of Cyp51 [35]. Thus, without entrainment of light, P450 genes still can keep oscillation under L-DD condition. Abnormal light influences xenobiotic metabolism and detoxification. Light signals induce transcription of heme oxygenase 2 and cytochrome P450 oxidoreductase [51]. Compared to LD conditions, mice under DD and LL reduce ethanol intake and ethanol preference [52]. Moreover, compared with LD condition recovery, constant darkness results in a faster recovery of both motor and anxiety impairments in alcohol

Table 2 Comparisons of hepatic cytochrome P450 genes under LD, S-DD and L-DD conditions

\begin{tabular}{|c|c|c|c|c|c|c|c|c|c|}
\hline \multirow[t]{2}{*}{ Name } & \multicolumn{3}{|c|}{$\boldsymbol{P}$-value } & \multicolumn{3}{|c|}{ Period (hour) } & \multicolumn{3}{|c|}{ rAmplitude } \\
\hline & $\overline{L D}$ & S-DD & L-DD & $\overline{L D}$ & S-DD & L-DD & $\mathrm{LD}$ & S-DD & L-DD \\
\hline & 0.14 & 0.83 & 0.05 & 23.59 & 25.33 & 23.02 & 0.19 & 0.15 & 0.39 \\
\hline yp2a4 & 0.0 & 0.04 & $0 . c$ & 4 & 2 & 20.34 & 1.21 & 9 & 0 \\
\hline 5 & 0.01 & 0.01 & 0.02 & 22.74 & 24.32 & 25.57 & 0.17 & 0.16 & 0.22 \\
\hline 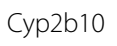 & 0.81 & 0.02 & 0.0 & 3 & 22.76 & 25.18 & 0.97 & 0.13 & 0 \\
\hline 9 & 0.50 & 0.87 & 0.03 & 2 & 21.33 & 25.34 & 0.01 & 0.05 & 0.05 \\
\hline yp2d40 & 0.01 & 0.94 & 0.03 & 25.26 & 24.00 & 20.28 & 0.06 & 0.06 & 0.07 \\
\hline 1 & 0.03 & 0.09 & 0.0 & 2 & 24.45 & 23.92 & 0.03 & 0.04 & 0. \\
\hline Cyp2g1 & 0.00 & 0.06 & 0.0 & 22.91 & 20.31 & 22.48 & 0.88 & 0.01 & 1.12 \\
\hline 1 & 0.66 & 0.11 & 0.02 & 2 & 20.23 & 20.34 & 0.16 & 0.05 & 0. \\
\hline Cyp51 & 0.15 & 0.57 & 0.02 & 20.51 & 21.33 & 25.33 & 0.09 & 0.04 & 0.06 \\
\hline yp7a1 & 0.82 & 0.05 & 0.04 & 21.33 & 21.33 & 22.86 & 0.13 & 0.13 & 0.15 \\
\hline
\end{tabular}

Note: 0.00 indicates that the values are less than 0.004 


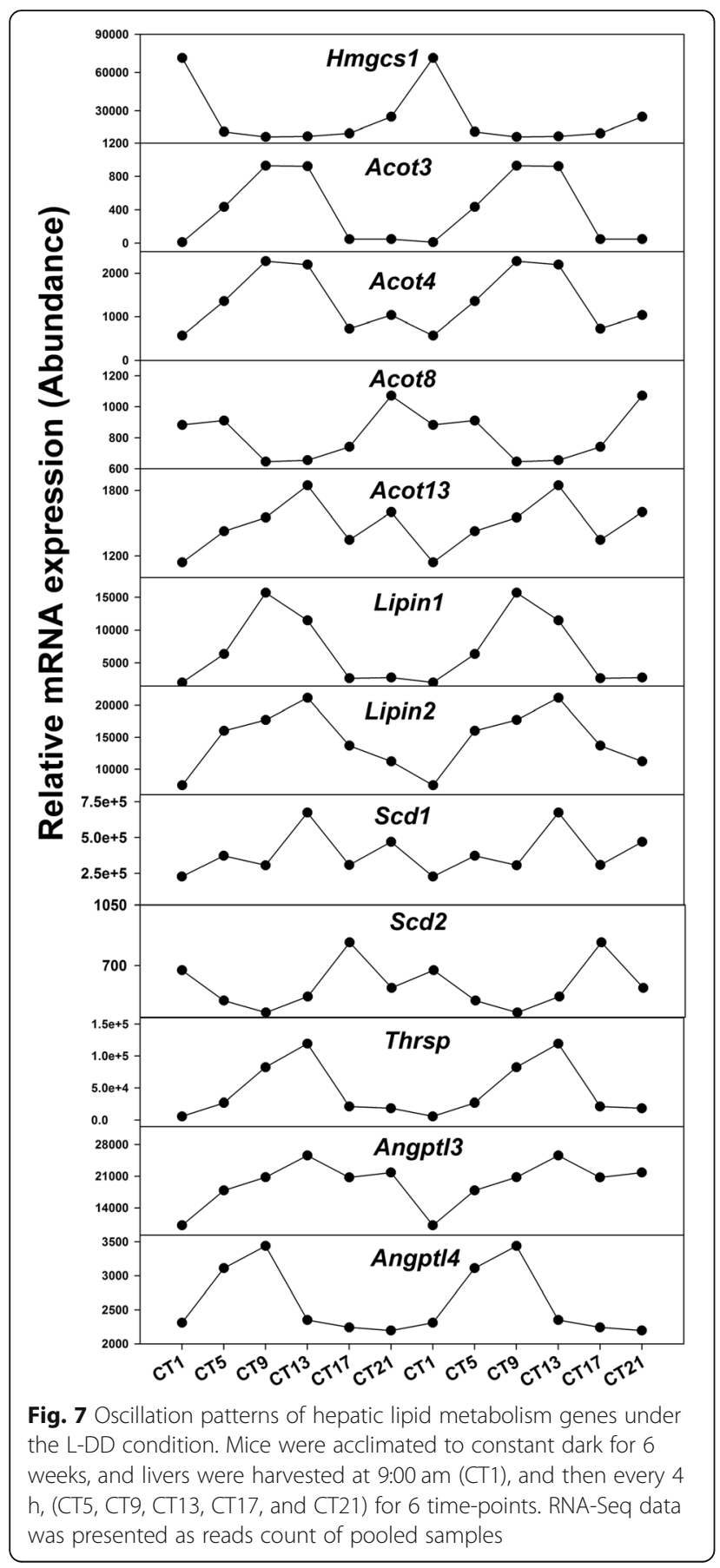

hangover swiss mice [53]. Profiling genes circadian rhythm under L-DD is important for metabolic study.

Cholesterol and fatty acids metabolism are regulated by the circadian clock $[11,54]$. The present study uncovered that some genes involved in cholesterol and lipid metabolism maintained rhythm under L-DD conditions (Fig. 7). The peak (nadir) of those oscillation genes under L-DD occurred at CT9 or CT13. The circadian rhythm of Acot3 and $A \cot 4$ are evidently induced by calorie restriction and are transcriptional targets of PPARa [55]. Compared to
LD, the circadian rhythm of $A \cot 3,4,8$ and 13 were also induced by L-DD with increased amplitude. Besides, the RT-qPCR also illustrated the oscillation of Acot3 and Acot4. It has been reported that Lipin1 has effects on both lipid synthesis and fatty acid oxidation to maintain lipid metabolic homeostasis [38]. Revealing its circadian rhythm under LD, S-DD and L-DD would help to understand the paradoxical efforts on lipid metabolism. The important lipogenic enzymes $S c d 1$ and $S c d 2$ are regulated by Bmal1 [56], and oscillate under LD and S-DD but not LDD. However, RT-qPCR results illustrated that there were strong daily fluctuations in the expression of $S c d 1$ and $S c d 2$. Our pervious study illustrated the circadian rhythm of Angptl8 [27]. The presented study further revealed the circadian rhythm of Angptl3 and Angptl4, which oscillated both under LD and L-DD. Abnormal light causes metabolic disorders. Our pervious study illustrated chronic exposure to green light aggravates high-fat diet-induced obesity and metabolic disorders in male mice [57]. L-DD is also thought to be a factor of light. Therefore, profiling circadian rhythm of cholesterol and lipid metabolism genes under L-DD would contribute to better understanding lipid metabolic homeostasis.

In Fig. 8, RT-qPCR was performed on genes of interest to further verify RNA sequence findings. Hmgcr is crucial in cholesterol metabolism and is the target of statin drugs to treat hypercholesterolemia [58]. Also, ACOTs constitute a family of enzymes that hydrolyze fatty acyl-CoAs to form FFA and CoA. Existing evidence suggests regulatory roles in controlling rates of peroxisomal and mitochondrial fatty acyl-CoA oxidation [37]. Scds are responsible for mitochondrial fatty acid $\beta$-oxidation of short chain fatty acids, and shift food consumption away from fat and toward carbohydrate, controlling lipid handling in the liver

Table 3 Comparisons of hepatic cholesterol and fatty acids metabolism genes under LD, S-DD and L-DD conditions

\begin{tabular}{|c|c|c|c|c|c|c|c|c|c|}
\hline \multirow[t]{2}{*}{ Name } & \multicolumn{3}{|c|}{$\boldsymbol{P}$-value } & \multicolumn{3}{|c|}{ Priod (hour) } & \multicolumn{3}{|c|}{ rAmplitude } \\
\hline & $\overline{L D}$ & $-D D$ & $\overline{D D}$ & $D$ & S-DD & L-DD & LD & S-DD & L-DD \\
\hline ngcs1 & 5 & n & 0.02 & 2 & A & 2 & 9 & NA & 3 \\
\hline 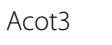 & .00 & .01 & 0.01 & 9 & 20.05 & 20.69 & 1.07 & 0.20 & 1.77 \\
\hline cot4 & 0.01 & 0.0 & 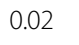 & 8 & 9 & 1 & 10 & 0 & 0.24 \\
\hline to & 35 & 0.16 & 0.02 & 87 & 22.89 & 20.37 & 0.04 & 0.03 & 0.06 \\
\hline & 0 & 0.11 & 0 & & 25.29 & 4 & 0.04 & 0.03 & 0.07 \\
\hline $\sin 1$ & 0.04 & 0.00 & 0.00 & 20.22 & 22.62 & 20.95 & 0.21 & 0.17 & 0.25 \\
\hline 年 & 0.02 & 0.01 & 0.0 & 3 & 12 & 2 & 0.08 & 0.0 & 0.10 \\
\hline 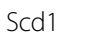 & 40 & 0.05 & 0.60 & 25.33 & 23.57 & 22.15 & 0.01 & 0.0 & 0. \\
\hline$d 2$ & (1) & 0.0 & 0.70 & 22.69 & 22.99 & & 0.05 & 0.07 & \\
\hline 1 & 0.45 & 0.06 & 0.00 & 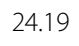 & 23. & 21.3 & 0.06 & 0.1 & 0. \\
\hline ingptl3 & 0.03 & 0.9 & 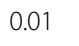 & 3 & 24.00 & 24.87 & 0.02 & 0.00 & 0 \\
\hline ngptl4 & 33 & 3 & 0. & .05 & 20 & 23.16 & 0.10 & 0.09 & 0 \\
\hline
\end{tabular}

Note: 0.00 indicates that the values are less than 0.004 


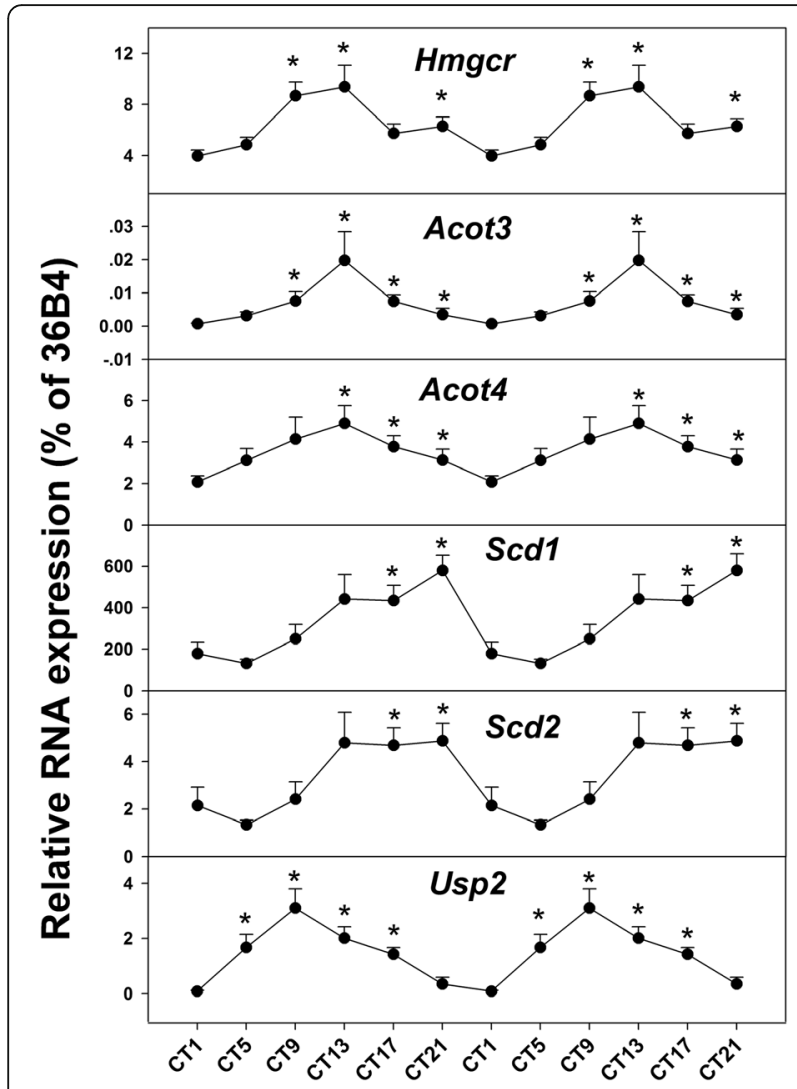

Fig. 8 RT-qPCR analysis of selected genes in the L-DD model. Mice were acclimated to constant dark for 6 weeks, and livers were harvested at 9:00 AM (CT1), and then every 4 h, (CT5, CT9, CT13, CT17, and CT21) for 6 time-points

[39]. For these selected genes, RT-qPCR verified their rhythms and confirmed the MetaCycle results.

\section{Conclusions}

In summary, the present gene profiling study revealed that under L-DD, endogenous circadian clock genes exhibited typical oscillation patterns, which drive the metabolic genes, including P450 genes and lipid metabolism genes in a diurnal variation pattern. Compared with LD and S-DD conditions, the oscillation patterns of these metabolism genes under L-DD were similar but the period and amplitude of those oscillation genes were slightly altered. These endogenous circadian rhythms are biological signals cross-talking with metabolism genes to maintain physiological functions.

\section{Methods}

\section{Animal treatment}

Six-week old SPF-grade male mice (C57BL/6 J) were purchased from Model Animal Research Center of Nanjing University (Nanjing, Jiangsu, China). The animal experiments were conducted according to the Guide for the Care and Use of Laboratory Animals published by the
National Institutes of Health (NIH publication) and approved by the Laboratory Animal Care Committee at China Pharmaceutical University (Permit number SYXK2018-0019). Mice were housed for 1-week acclimation under a 12:12-h light/dark cycle, controlled temperature $\left(22-24{ }^{\circ} \mathrm{C}\right)$ and humidity $(50-60 \%)$ with free access to water and standard rodent chaw (normal diets with $4 \%$ of energy from lipid, XieTong Organism, China).

Subsequently, 42 mice were randomly divided into six groups, and each group had 7 mice, with 3-4 mice per cage. The animals were kept in constant dark condition (DD) for 6 weeks to eliminate bright light influence before experiments. Mice were sacrificed by dislocation of the cervical vertebrae. Livers were collected starting from 9:00 AM (CT1), and then every $4 \mathrm{~h}$ (13:00; 17:00; 21:00; 1:00; 5:00), corresponding to CT5, CT9, CT13, CT17 and CT21.

\section{Transcriptome sequencing}

The liver samples were homogenized, and total RNA was extracted using TRIzol reagent (Molecular Research Center, USA). RNA quality and quantity were determined with Agilent 2200 TapeStation (Agilent Technologies, USA), with $260 / 280>1.8$, and $260 / 230>1.4$ as a quality reference. Pooled samples from 7 mice at each time point were sent to Guangzhou RiboBio Co., Ltd. (Guangzhou, China) for RNA sequencing, using Illumina HiSeq3000. The cDNA libraries were constructed with the Illumina Sample Preparation protocol (Illumina, San Diego, CA) to generate 100-bp paired-end reads. The raw sequencing data (FASTQ files) was aligned to the mouse genome (NCB137/mm10) using Bowtie. The RNA-seq data under 6 weeks of constant darkness (called long time dark-dark condition, L-DD) was deposited, and the GEO number is GSE133342 [27]. To compare gene oscillation pattern under normal light-dark condition (LD) and short time (2 days) dark-dark condition (S-DD), the data were retried from GEO database for LD (GSE114400, [28]) and S-DD (GSE70497, [29]).

\section{Normalization and oscillation genes identification}

To avoid the differences of sequencing depths from different libraries, the method TMM was used to normalize the three RNA-sequences through $\mathrm{R}$ (64-bit, version 3.6.1) package "EdgeR" [59]. After the normalization, MetaCycle, an R package that incorporates ARSER, JTK_CYCLE and Lomb-Scargle to conveniently evaluate periodicity in timeseries data, was performed to identify oscillation genes [60]. The output result of MetaCycle contained $p$-value, period, and revised amplitude. Oscillating transcripts were defined as $P<0.05$. Heatmaps were generated using the pheatmap package to display the oscillation genes. The Venn Diagram Plotter was used to compare the rhythmic genes under LD, 
S-DD and L-DD and generated by VennDiagram package [61].

\section{Gene ontology (GO) and Kyoto encyclopedia of genes and genomes (KEGG) analysis}

To understand biological function of the rhythmic genes under LD, S-DD and L-DD, GO (Biological process) and KEGG pathways were performed using $\mathrm{R}$ package clusterProfiler [62]. A value of $P<0.05$ was used as a cutoff for significantly enriched terms. The graphs were drawn by R package Enrichplot [63].

\section{RT-qPCR analysis}

Total RNA was reverse transcribed to cDNA with TakaRa RT kits (Takara Bio, Japan) according to manufacture instructions. The $10 \mu \mathrm{L}$ PCR reaction mix contained $2 \mu \mathrm{L}$ of cDNA $(10 \mathrm{ng} / \mathrm{ml}), 5 \mu \mathrm{L}$ qPCR SYBR mix (Takara Bio, Japan), $0.3 \mu \mathrm{L}$ of primer mix ( $25 \mu \mathrm{M}$ each) and $2.7 \mu \mathrm{L}$ of $\mathrm{ddH}_{2} \mathrm{O}$. The Real-time qPCR cycler (Roche Light Cycler 480 II) program was $5 \mathrm{~min}$ at $95^{\circ} \mathrm{C}, 40 \mathrm{cy}$ cles of annealing and extension at $60^{\circ} \mathrm{C}$ for $30 \mathrm{~s}$ and denaturation at $95^{\circ} \mathrm{C}$ for $10 \mathrm{~s}$. Dissociation curve was performed after the 40 cycles are over to verify the quality of primers and amplification. The values obtained were used to calculate the expression of clock genes by the $2^{-\triangle \Delta C t}$ method and normalized to the housekeeping gene 36B4. The relative transcript levels were calculated setting the control as $100 \%$.

\section{Statistical analysis}

The RT-qPCR data was presented as group mean with SEM $(n=7)$. Statistical analysis was completed using Statistical Package for the Social Sciences (SPSS) software (version 17.0). Before statistical analysis, all the data were inserted into the software to guarantee they followed normal distributions. Student's t test was used to compare the differences between CT1 and the other time points. $P<$ 0.05 was considered to indicate a statistical significance.

\section{Supplementary information}

Supplementary information accompanies this paper at https://doi.org/10. $1186 /$ s12864-020-6639-4.

Additional file 1. Oscillation genes under L-DD. RNA-Seq data of L-DD was subjected to MetaCycle to screen oscillation genes.

Additional file 2. Oscillation genes under LD. RNA-Seq data of LD was subjected to MetaCycle to screen oscillation genes.

Additional file 3. Oscillation genes under S-DD. RNA-Seq data of S-DD was subjected to MetaCycle to screen oscillation genes.

\section{Abbreviations}

Acot3,4,8,13: Acyl-CoA thioesterase3,4,8,13; Angpt13,4: Angiopoietin like 3,4; CT: Circadian time; Cyps: Cytochrome P450 family genes; DD: Constant darkness; GO: Gene ontology; KEGG: Kyoto encyclopedia of genes and genomes; LL: Constant light; Scd1,Scd2: Stearoyl-Coenzyme A desaturase1, 2;
SCN: Hypothalamic suprachiasmatic nucleus; TMM: Trimmed mean of Mvalues normalization method; ZT: Zeitgeber time

\section{Acknowledgements}

We thank Drs. Johnstone Andrew F (US EPA) and David Chen (NIEHS) for critical review of the manuscript.

\section{Authors' contributions}

HL contributed to conception and design of experiments, data acquisition, analysis and interpretation of data, and was involved in drafting and writing the manuscript. SYZ, SYC and WXZ contributed to data acquisition and analysis and interpretation of data. $J$ and JCC contributed to analysis and interpretation of data and was involved in revising the manuscript. AR and YJS contributed to analysis and interpretation of data and was involved in revising the manuscript. $C L$ contributed to conception and design of experiments and revising the manuscript. All authors read and approved the final manuscript.

\section{Funding}

This study was supported by the National Natural Science Foundation of China (Nos. 31800992, 31771298, 81800512), Postgraduate Research \& Practice Innovation Program of Jiangsu Province (KYCX18_0781, KYCX19 0660). The funding agency had no role in the design of the study, collection, analysis, or interpretation of data, or in writing the manuscript.

\section{Availability of data and materials}

Three sets of RNA-seq data (Accession number: GSE114400, GSE70497, GSE133342) generated and/or analyzed during the current study are available in the GEO repository, https://www.ncbi.nlm.nih.gov/geo/query/acc.cgi.

\section{Ethics approval and consent to participate}

The animal experiments were conducted according to the Guide for the Care and Use of Laboratory Animals published by the National Institutes of Health (NIH publication) and approved by the Laboratory Animal Care Committee at China Pharmaceutical University (Permit number SYXK-20180019)

Consent for publication

Not applicable.

\section{Competing interests}

The authors declare that they have no competing interests.

\section{Author details}

${ }^{1}$ School of Life Sciences and Technology, China Pharmaceutical University, Nanjing, China. ${ }^{2}$ Key Laboratory of Basic Pharmacology of Ministry of Education, Zunyi Medical University, Zunyi, Guizhou, China. ${ }^{3}$ Computational Toxicology Division, US Environmental Protection Agency, Research Triangle Park, NC, USA.

Received: 12 September 2019 Accepted: 3 March 2020

Published online: 12 March 2020

\section{References}

1. Pittendrigh CS. Temporal organization: reflections of a Darwinian clockwatcher. Annu Rev Physiol. 1993;55:16-54

2. Panda S. Circadian physiology of metabolism. Science. 2016;354(6315): 1008-15.

3. Mohawk JA, Green CB, Takahashi JS. Central and peripheral circadian clocks in mammals. Annu Rev Neurosci. 2012;35(1):445-62.

4. Dibner $\mathrm{C}$, Schibler $\mathrm{U}$, Albrecht $\mathrm{U}$. The mammalian circadian timing system: organization and coordination of central and peripheral clocks. Annu Rev Physiol. 2010;72(1):517.

5. Buhr ED, Takahashi JS. Molecular Components of the Mammalian Circadian Clock. Handb Exp Pharmacol. 2013;15 Spec No 2(217):3.

6. Takahashi JS. Transcriptional architecture of the mammalian circadian clock. Nat Rev Genet. 2017;18(3):164-79.

7. Partch $\mathrm{CL}$, Green CB, Takahashi JS. Molecular architecture of the mammalian circadian clock. Trends Cell Biol. 2014;24(2):90-9.

8. Reinke H, Asher G. Circadian clock control of liver metabolic functions. Gastroenterology. 2016;150(3):574-80. 
9. Feng D, Lazar MA. Clocks, metabolism, and the epigenome. Mol Cell. 2012 47(2):158-67.

10. Reinke H, Asher G. Crosstalk between metabolism and circadian clocks. Nat Rev Mol Cell Biol. 2019;20(4):227-41.

11. Guan D, Xiong Y, Borck PC, Jang C, Doulias P-T, Papazyan R, Fang B, Jiang C, Zhang $Y$, Briggs ER, et al. Diet-Induced Circadian Enhancer Remodeling Synchronizes Opposing Hepatic Lipid Metabolic Processes. Cell. 2018;174(4): 831-42 e812.

12. Turek FW, Corinne J, Akira K, Emily L, Ganka I, Erin MD, Aaron L, Sue LO, Amy E, Jensen DR. Obesity and metabolic syndrome in circadian Clock mutant mice; 2005.

13. Zhang EE, Liu Y, Dentin RPongsawakul PY, Liu AC, Hirota T, Nusinow DA, Sun X, Landais S, Kodama Y, Brenner DA. Cryptochrome mediates circadian regulation of CAMP signaling and hepatic gluconeogenesis. Nat Med. 2010; 16(10):1152-6.

14. Duez H, Van-Der-Veen J, Duhem C, Pourcet B, Touvier T, Fontaine C, Derudas B, Bauge E, Havinga R, Bloks V, Wolters H. Regulation of bile acid synthesis by the nuclear receptor Rev-erbalpha. Gastroenterology. 2008; 135(2):689-98 e685.

15. Golombek DA, Rosenstein RE. Physiology of circadian entrainment. Physiol Rev. 2010;90(3):1063-102

16. Colwell CS. 3. The Suprachiasmatic Nucleus (SCN); 2015.

17. Jud C, Schmutz I, Hampp G, Oster H, Albrecht U. A guideline for analyzing circadian wheel-running behavior in rodents under different lighting conditions. Biol Proced Online. 2005;7:101-16.

18. Lee CC. Constant darkness is a mammalian biological signal. Cold Spring Harb Symp Quant Biol. 2007;72:287-91.

19. Ueda HR, Chen W, Adachi A, Wakamatsu H, Hayashi S, Takasugi T, Nagano M, Nakahama K, Suzuki Y, Sugano S, et al. A transcription factor response element for gene expression during circadian night. Nature. 2002;418(6897): 534-9.

20. Mei L, Fan Y, Lv X, Welsh DK, Zhan C, Zhang EE. Long-term in vivo recording of circadian rhythms in brains of freely moving mice. Proc Natl Acad Sci U S A. 2018;115(16):4276-81.

21. Nakamura TJ, Nakamura W, Tokuda IT, Ishikawa T, Kudo T, Colwell CS, Block GD. Age-Related Changes in the Circadian System Unmasked by Constant Conditions. eNeuro. 2015;2(4):0064-15.

22. Zhang J, Kaasik K, Blackburn MR, Lee CC. Constant darkness is a circadian metabolic signal in mammals. Nature. 2006;439(7074):340-3.

23. Goff ML, Finger FW. Activity rhythms and adiurnal light-dark control. Science (New York, NY). 1966;154(3754):1346-9.

24. Yassumoto TI, Tachinardi P, Oda GA, Valentinuzzi VS. Acute effects of light and darkness on the activity and temperature rhythms of a subterranean rodent, the Anillaco tuco-tuco. Physiol Behav. 2019;210:112645.

25. Zhou Y, Zhang HK, Liu F, Lei G, Liu P, Jiao T, Dang YH. Altered light conditions contribute to abnormalities in emotion and cognition through HINT1 dysfunction in C57BL/6 mice. Front Behav Neurosci. 2018;12:110.

26. Kim YM, Snijders AM, Brislawn CJ, Stratton KG, Zink EM, Fansler SJ, Metz TO, Mao JH, Jansson JK. Light-stress influences the composition of the murine gut microbiome, memory function, and plasma Metabolome. Front Mol Biosci. 2019;6:108.

27. Chen S, Feng M, Zhang S, Dong Z, Wang Y, Zhang W, Liu C. Angptl8 mediates food-driven resetting of hepatic circadian clock in mice. Nat Commun. 2019;10(1):3518.

28. Weger BD, Gobet C, Yeung J, Martin E, Jimenez S, Betrisey B, Foata F, Berger B, Balvay A, Foussier A, et al. The Mouse Microbiome Is Required for SexSpecific Diurnal Rhythms of Gene Expression and Metabolism. Cell metabolism. 2019;29(2):362-82 e368.

29. Yang G, Chen L, Grant GR, Paschos G, Song W-L, Musiek ES, Lee V, McLoughlin SC, Grosser T, Cotsarelis G, et al. Timing of expression of the core clock gene Bmal1 influences its effects on aging and survival. Sci Transl Med. 2016:8(324):324ra316.

30. Zanger UM, Schwab M. Cytochrome P450 enzymes in drug metabolism: regulation of gene expression, enzyme activities, and impact of genetic variation. Pharmacol Ther. 2013;138(1):103-41.

31. Almazroo OA, Miah MK, Venkataramanan R. Drug metabolism in the liver. Clinics Liver Dis. 2017;21(1):1-20.

32. Froy O. Cytochrome P450 and the biological clock in mammals. Current Drug Metabolism. 2009;10(2):104-15.

33. Yuan-Fu L, Tao J, Yasha X, Dan Z, Qin W, Yu-Kun Jennifer Z, Jie L. Sex differences in the circadian variation of cytochrome p450 genes and corresponding nuclear receptors in mouse liver. Chronobiol Int. 2013;30(9): 1135-43.

34. Chambers KF, Day PE, Aboufarrag HT, Kroon PA. Polyphenol effects on cholesterol metabolism via bile acid biosynthesis, CYP7A1: a review. Nutrients. 2019;11(11):2588

35. Acimovic J, Fink M, Pompon D, Bjorkhem I, Hirayama J, Sassone-Corsi P, Golicnik M, Rozman D. CREM modulates the circadian expression of CYP51, HMGCR and cholesterogenesis in the liver. Biochem Biophys Res Commun. 2008;376(1):206-10.

36. Maxwell KN, Soccio RE, Duncan EM, Sehayek E, Breslow JL. Novel putative SREBP and LXR target genes identified by microarray analysis in liver of cholesterol-fed mice. J Lipid Res. 2003;44(11):2109-19.

37. Tillander V, Alexson SEH, Cohen DE. Deactivating fatty acids: acyl-CoA Thioesterase-mediated control of lipid metabolism. Trends Endocrinol Metab. 2017;28(7):473-84.

38. Chen Y, Rui BB, Tang LY, Hu CM. Lipin family proteins--key regulators in lipid metabolism. Ann Nutr Metab. 2015;66(1):10-8.

39. Ghosh S, Kruger C, Wicks S, Simon J, Kumar KG, Johnson WD, Mynatt RL, Noland RC, Richards BK. Short chain acyl-CoA dehydrogenase deficiency and short-term high-fat diet perturb mitochondrial energy metabolism and transcriptional control of lipid-handling in liver. Nutr Metab. 2016;13:17.

40. Li J, Li L, Guo D, Li S, Zeng Y, Liu C, Fu R, Huang M, Xie W. Triglyceride metabolism and angiopoietin-like proteins in lipoprotein lipase regulation. Clin Chim Acta. 2020;503:19-34..

41. Li J, Grant GR, Hogenesch JB, Hughes ME. Considerations for RNA-seq analysis of circadian rhythms. Methods Enzymol. 2015;551:349-67.

42. Schwartz WJ, Daan S. Origins: a brief account of the ancestry of circadian biology. In: Biological timekeeping: clocks, rhythms and behaviour. Springer (India) Pvt. Ltd. 2017:3-22.

43. Cai M, Liu Z, Yu P, Jiao Y, Chen Q, Jiang Q, Zhao Y. Circadian rhythm regulation of the oxidation-antioxidant balance in Daphnia pulex. Comp Biochem Physiol B Biochem Mol Biol. 2019;240:110387.

44. Ali AAH, Stahr A, Ingenwerth M, Theis M, Steinhauser C, von Gall C. Connexin30 and Connexin43 show a time-of-day dependent expression in the mouse suprachiasmatic nucleus and modulate rhythmic locomotor activity in the context of chronodisruption. Cell Commun Signal. 2019;17(1):61.

45. Spoelstra K, Oklejewicz M, Daan S. Restoration of self-sustained circadian rhythmicity by the mutant clock allele in mice in constant illumination. J Biol Rhythm. 2002;17(6):520-5.

46. Zheng B, Albrecht U, Kaasik K, Sage M, Lu W, Vaishnav S, Li Q, Sun ZS, Eichele G, Bradley A, et al. Nonredundant roles of the mPer1 and mPer2 genes in the mammalian circadian clock. Cell. 2001;105(5):683-94.

47. Cho H, Zhao X, Hatori M, Yu RT, Barish GD, Lam MT, Chong L-W, DiTacchio $L$, Atkins AR, Glass CK, et al. Regulation of circadian behaviour and metabolism by REV-ERB- $a$ and REV-ERB-B. Nature. 2012;485(7396):123-7.

48. Kosir R, Spaninger K, Rozman D. Circadian events in human diseases and in cytochrome P450-related drug metabolism and therapy. IUBMB Life. 2013; 65(6):487-96.

49. Froy O. Cytochrome P450 and the biological clock in mammals. Curr Drug Metab. 2009;10(2):104-15.

50. Takeda Y, Kang HS, Lih FB, Jiang H, Blaner WS, Jetten AM. Retinoid acidrelated orphan receptor gamma, RORgamma, participates in diurnal transcriptional regulation of lipid metabolic genes. Nucleic Acids Res. 2014; 42(16):10448-59.

51. Ben-Shlomo R, Akhtar RA, Collins BH, Judah DJ, Davies R, Kyriacou CP. Light pulse-induced heme and iron-associated transcripts in mouse brain: a microarray analysis. Chronobiol Int. 2005;22(3):455-71.

52. Rosenwasser AM, McCulley WD 3rd, Hartmann MC, Fixaris MC, Crabbe JC. Suppression of Voluntary Ethanol Intake in Mice under Constant Light and Constant Darkness. Alcohol. 2019;S0741-8329(0718):30295-7.

53. Karadayian AG, Lores-Arnaiz S, Cutrera RA. The effect of constant darkness and circadian resynchronization on the recovery of alcohol hangover. Behav Brain Res. 2014;268(4):94-103.

54. Gorné LD, Acosta-Rodríguez VA, Pasquaré SJ, Salvador GA, Giusto NM, Mario Eduardo $G$. The mouse liver displays daily rhythms in the metabolism of phospholipids and in the activity of lipid synthesizing enzymes. Chronobiol Int. 2015;32(1):11-26.

55. Makwana K, Gosai N, Poe A, Kondratov RV. Calorie restriction reprograms diurnal rhythms in protein translation to regulate metabolism. FASEB J. 2019;33(3):4473-89. 
56. Zhang D, Tong X, Arthurs B, Guha A, Rui L, Kamath A, Inoki K, Yin L. Liver clock protein BMAL1 promotes de novo lipogenesis through insulinmTORC2-AKT signaling. J Biol Chem. 2014;289(37):25925-35.

57. Zhang S, Zhang Y, Zhang W, Chen S, Liu C. Chronic exposure to green light aggravates high-fat diet-induced obesity and metabolic disorders in male mice. Ecotoxicol Environ Saf. 2019;178:94-104.

58. Borthwick F, Mangat R, Warnakula S, Jacome-Sosa M, Vine DF, Proctor SD. Simvastatin treatment upregulates intestinal lipid secretion pathways in a rodent model of the metabolic syndrome. Atherosclerosis. 2014;232(1):141-8

59. Pereira MB, Wallroth M, Jonsson V, Kristiansson E. Comparison of normalization methods for the analysis of metagenomic gene abundance data. BMC Genomics. 2018;19(1):274.

60. Wu G, Anafi RC, Hughes ME, Kornacker K, Hogenesch JB. MetaCycle: an integrated $R$ package to evaluate periodicity in large scale data. Bioinformatics. 2016;32(21):3351-3.

61. Chen H, Boutros PC. VennDiagram: a package for the generation of highlycustomizable Venn and Euler diagrams in R. BMC Bioinformatics. 2011;12:35.

62. Yu G, Wang L-G, Han Y, He Q-Y. clusterProfiler: an R package for comparing biological themes among gene clusters. OMICS. 2012;16(5):284-7.

63. Yu G. enrichplot: Visualization of Functional Enrichment Result. R package version $112 ; 2018$

\section{Publisher's Note}

Springer Nature remains neutral with regard to jurisdictional claims in published maps and institutional affiliations.

Ready to submit your research? Choose BMC and benefit from:

- fast, convenient online submission

- thorough peer review by experienced researchers in your field

- rapid publication on acceptance

- support for research data, including large and complex data types

- gold Open Access which fosters wider collaboration and increased citations

- maximum visibility for your research: over $100 \mathrm{M}$ website views per year

At $\mathrm{BMC}$, research is always in progress.

Learn more biomedcentral.com/submissions 